\title{
Recognising Activities in Real Time Using Body Worn Passive Sensors With Sparse Data Streams: To Interpolate or Not To Interpolate?
}

\author{
Asanga Wickramasinghe \\ AutolD Lab, University of Adelaide \\ Adelaide, Australia \\ asanga.wickramasinghe@adelaide.edu.au
}

\author{
Damith C. Ranasinghe \\ AutolD Lab, University of Adelaide \\ Adelaide, Australia \\ damith.ranasinghe@adelaide.edu.au
}

\begin{abstract}
Recent emergence of small, lightweight, batteryless (passive), and therefore maintenance free, wearable computing platforms such as sensor enabled RFID (Radio Frequency Identification) tags provide new opportunities for low cost and unobtrusive activity monitoring. Unfortunately, data streams from passive sensors are uniquely characterised by sparsity and noise. Consequently, readily extracting features that require a data stream with a regular sampling rate, such as those based on frequency domain transformations and auto regressive coefficients are challenging. In this study, we propose an approach that reduces online interpolation errors to facilitate interpolating sparse acceleration data streams from a passive RFID tag with an on-board accelerometer sensor. We demonstrate using two datasets from older people that incorporating features extracted from interpolated acceleration data depicts a significant performance improvement compared with incorporating features possible from only raw acceleration data. However, this is achieved at the expense of a real-time prediction delay $(>400 \%)$ due to preprocessing involved. Furthermore, we demonstrate that for these types of sensors, features readily available from a typical RFID platform can be successfully used instead of features extracted from an interpolated data stream to achieve similar or better activity recognition performance without preprocessing and, whilst, using significantly less number of features.
\end{abstract}

\section{Categories and Subject Descriptors}

I.5.2 [Pattern Recognition]: Design Methodology-Pattern analysis; I.5.4 [Pattern Recognition]: ApplicationsSignal processing; I.5.m [Pattern Recognition]: miscellaneous-RFID based activity recognition

\section{General Terms}

Experimentation, Algorithms

Permission to make digital or hard copies of all or part of this work for personal or classroom use is granted without fee provided that copies are not made or distributed for profit or commercial advantage and that copies bear this notice and the full citation on the first page. To copy otherwise, to republish, to post on servers or to redistribute to lists, requires prior specific permission and/or a fee. MOBIQUITOUS 2015, July 22-24, Coimbra, Portugal Copyright $\odot 2015$ ICST 978-1-63190-072-3

DOI 10.4108/eai.11-8-2015.151111

\section{Keywords}

Wearable passive rfid tags; Sparse acceleration data streams; Interpolation; Human activity recognition; Features

\section{INTRODUCTION}

Automatic identification of human activities using wearable sensors is a growing field of study that forms the basis for many ubiquitous healthcare applications such as gait analysis 25,31, rehabilitation 26 and falls risk activity recognition 28, 41]. Most activity recognition (AR) studies based on body-worn sensors use bulky, battery powered devices strapped or attached to various parts of the participants body such as thighs 24, 41, arms [16. and the head 42. Some of the studies even utilize multiple sensors such as accelerometers and gyroscopes attached to multiple body locations 3, 10, 36. Use of these types of bulky and obtrusive sensors for monitoring, particularly older people is unsuitable as reported by user acceptability studies where unobtrusiveness is highlighted as one of the key acceptance criteria 13. Furthermore, these types of sensors also require maintenance such as regular recharging or replacement of batteries during their operational life.

In contrast, a new generation of passive (batteryless) sensors such as sensor enabled RFID (Radio Frequency Identification) tags 18, 32 are creating exciting new prospects for wearable sensor based applications. As opposed to battery powered sensors, passive sensors are lightweight and small, hence they can be used for unobtrusive monitoring. In addition, passive sensors are maintenance free as they require no battery. Furthermore, these sensors can be easily embedded into garments and thereby preventing removal of the monitoring device, specially by cognitively impaired patients 23 . Therefore, technological solutions for monitoring human activities of older people based on passive sensors are more likely to be accepted in practice 13,34 .

In this study, we focus on the use of a single passive sensor enabled RFID tag embedded with an accelerometer (henceforth sensor tag) for real-time AR. Apart from the acceleration signal, sensor enabled RFID tags also provide information related to signal strength (see Section 3.1] 34, which provide contextual information.

However, unlike battery powered sensors, data streams from passive sensors are noisy, and sparse with low and variable sampling rates because passive sensors need to har- 
vest adequate energy prior to powering and sampling a sensor 32,34 . As a result of this sparsity, features that require a data stream with a regular sampling rate cannot readily be extracted, particularly for machine learning based AR. For example, frequency-domain features [2, 3, 24, which constitute a significant number of features used in machine learning based activity recognition studies, require a data stream with a regular sampling rate. More recently, autoregressive coefficients have successfully been used as features in AR 20]; these features also require a data stream with a regular sampling rate. Common solution to address the sparsity is the application of interpolation methods to resample the sensor data stream to achieve a regular sampling rate. Although, there are methods to obtain frequencydomain features based on Non Uniform Fast Fourier Transform (NUFFT) from sparse data streams [8, 12], other features such as autoregressive coefficients and discrete cosine transformation coefficients 15,20 that requires a regular sampling rate cannot be extracted using these approaches.

In this paper, we investigate whether additional features possible from interpolated acceleration signals from passive sensor enable RFID tags would yield a performance improvement over the use of a limited set of time domain features constructed using biomechanical analysis from sparse acceleration signals for real-time AR. To ground our work, we have selected two real-world datasets collected for ambulatory monitoring of older patients in patients' rooms 28,29$]$. The main contributions of this study are:

- We propose a dynamic sensor data augmentation algorithm, an approach to facilitate online interpolation of sparse acceleration data streams from passive sensors to allow extraction of features that require a data stream with regular sampling rate. Our approach reduces interpolation errors when interpolating sparse sensor observations that are temporally distant by augmenting the sensor data stream using most recent sensor observations.

- We implement five real-time sensor data stream interpolation methods with increasing order of complexity. We evaluate activity recognition performance and time taken to interpolate streaming sensor data collected from older volunteers (66-86 years) wearing a passive sensor enabled RFID tag embedded with an accelerometer over their attire in a clinical environment.

- We demonstrate that when only acceleration signals are considered, set of additional features from interpolated acceleration data (auto regressive features and frequency-domain features) significantly improve activity recognition performance over time-domain features based on biomechanical studies of human movements obtained from sparse acceleration data.

- We also show that, in general, features generated through information available from an RFID platform, as in our case, can effectively be used for activity recognition instead of additional features, i.e. auto regressive features and frequency-domain features, to achieve similar performance without additional preprocessing.
Following sections of the paper are organized as follows: Section 2 discusses related works; Section 3 presents the passive sensor, the data set used for the evaluation and discusses the interpolation methods and features; Section 4 presents our evaluation and results; and we conclude the paper in Section 5

\section{RELATED WORKS}

To date, number of studies that focus on real-time activity

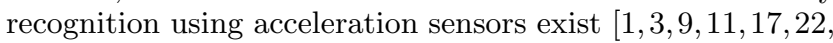

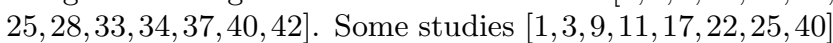
are based on battery powered sensors while recent studies use passive sensors \begin{tabular}{ll|l|l|l|l}
2 & 28 & 29 & 33 & 34 & . In these works human ac-
\end{tabular} tivities are recognized based on empirically determined decision tree algorithms or using machine learning algorithms to automatically learn the activity patterns embedded in acceleration data streams.

Activity recognition research based on machine learning techniques have extracted number of features from acceleration signals and they can be categorized as: i) time-domain features; ii) frequency-domain features; and iii) features based on biomechanics and heuristics 10. Features such as mean, standard deviation, variance of the acceleration signal were used as time-domain features 3,10 . Frequency-domain features include Fast Fourier Transformation (FFT) coefficients 24], Discrete Cosine Transformation Coefficients [15], spectral energy 3. and entropy 3. Features such as trunk

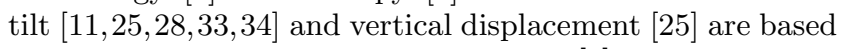
on biomechanics. Inter axes correlation [3] and coefficients of an auto regressive model 20 are examples of features that are based on heuristics. Majority of features for machine learning base AR are frequency-domain based features. Calculating frequency-domain features require a signal with a regular sampling rate. Furthermore, calculating features such as auto regressive coefficients [20], also requires a data stream with regular sensor observation.

The sparsity of the data streams from passive sensors reduces the number of features that can be readily calculated from raw acceleration signals without further processing and consequently incurring additional delays. Therefore, we are motivated to investigate whether additional features based on interpolated acceleration signals from passive sensor enabled RFID tags can yield a performance improvement over the use of a limited set of time-domain features from sparse acceleration signals for activity recognition. Specifically, we consider frequency-domain features based on FFT and auto regressive coefficients from interpolated acceleration signals as learning with these features have resulted in highly accurate activity prediction models in previous studies [20,42.

\section{METHODOLOGY}

\subsection{Sensor Tag}

We utilized a passive (batteryless) sensor enabled RFID tag called Wearable Wireless Identification and Sensing Platform (W ${ }^{2} \mathrm{ISP}$ ) tag (Fig. 1) 18, which is based on the Wireless Identification and Sensing Platform (WISP) 32], embedded with a 3D acceleration sensor (ADXL330). Thus, this sensing device act as a passive acceleration sensor.

Like any passive RFID tag, $\mathrm{W}^{2}$ ISP harvests power from radiation emitted by the RFID reader antennas and read by a 


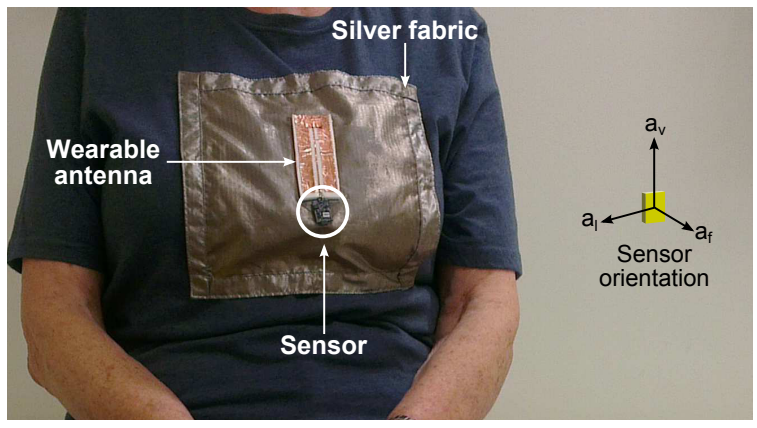

Figure 1: A participant wearing the $\mathrm{W}^{2}$ ISP at the sternum level over the garment.

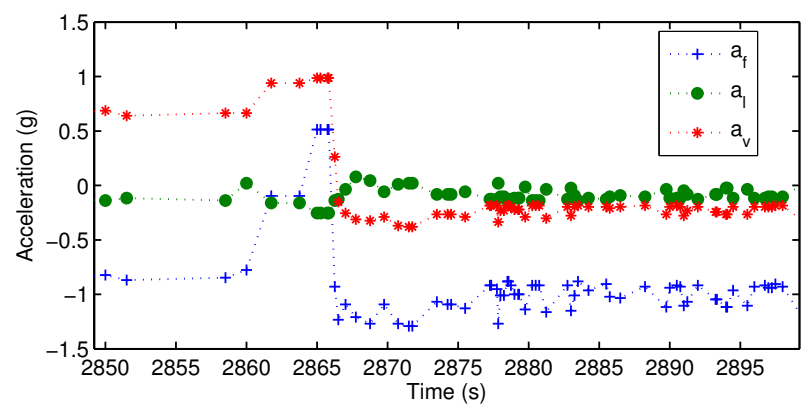

Figure 2: A typical data stream obtained using the sensor tag illustrating sparsity and noise

standard off-the-shelf UHF (Ultra High Frequency) RFID reader where sensor data is embedded in the tag identifier 35 . W $\mathrm{W}^{2} \mathrm{ISP}$ can be read approximately from $4 \mathrm{~m}$ when worn by a human, weighs approximately $3 \mathrm{~g}$ and mass production cost per tag is estimated to be about $\$ 3[5]$. When a $\mathrm{W}^{2} \mathrm{ISP}$ is adequately powered, a data stream with an upper bound of $40 \mathrm{~Hz}$ sampling rate can be obtained.

However, successful powering of the $\mathrm{W}^{2} \mathrm{ISP}$ depends on several factors. A decrease in the strength of radiation from RFID reader antennas due to: i) $\mathrm{W}^{2}$ ISP being further away from antennas; ii) occlusion of radio signals from radio frequency opaque objects such as a human body; and iii) change in $\mathrm{W}^{2}$ ISP antenna orientation with respect to RFID reader antennas due to body motion leading to less power being harvested. Power harvesting is also hindered by signal cancellation at the $\mathrm{W}^{2}$ ISP due to multipath effect and radio signal interference from other devices that uses the same radio spectrum (920-926 MHz in Australia) 33. Therefore, sensor data streams from $\mathrm{W}^{2} \mathrm{ISP}$, and generally from passive sensors and sensor enabled RFID tags are: i) noisy due to inadequate power to sample the embedded physical sensor; and ii) sparse (variable inter sensor observation time intervals) with low sampling rate (sensor observations per second). Figure 2 shows portions of acceleration measurements obtained during data collection (see Section 3.2 using the sensor tag, where it clearly shows the data stream characteristics, i.e. sparsity and noise. For instance, during $2850 \mathrm{~s}$ to 2860 s period only 3 data points are available where as from $2885 \mathrm{~s}$ to $2895 \mathrm{~s}$ there are 16 data points.

We obtain the 5-tuple $\left[a_{f}, a_{l}, a_{v}, R S S I, a I D\right]$ from each sen-
Table 1: Distribution of activities in data sets.

\begin{tabular}{|c|c|c|c|c|}
\hline & Sitting on bed & Lying on bed & Ambulation & Sitting on chair \\
\hline Roomset1 & $15162(25.89 \%)$ & $30983(59.04 \%)$ & $1956(3.73 \%)$ & $4381(8.35 \%)$ \\
\hline Roomset2 & $1253(5.53 \%)$ & $20529(90.65 \%)$ & $334(1.47 \%)$ & $530(2.34 \%)$ \\
\hline
\end{tabular}

sor observation. Here, $a_{f}, a_{l}$ and $a_{v}$ represent frontal, lateral and vertical accelerations measured with reference to the acceleration sensor (Fig.1) respectively, RSSI (Received Signal Strength Indicator) represents the power of the radio signal of a sensor observation sent by the sensor and received by a specific antenna and measured by an RFID reader, and $a I D$ represents the identifier of the antenna that captured the observation. Thus, we have information not only from an accelerometer, but also from the RFID platform.

\subsection{Data Set}

We used two data sets described in 33,34 that are collected for ambulatory monitoring of older patients to conduct our experiments. These data sets include activities: i) sitting on bed; ii) lying on bed; iii) ambulation; and iv) standing. These data sets were collected in two clinical room configurations (Roomset1 and Roomset2) (Fig. 3). Roomset1 configuration was equipped with four RFID reader antennas, and one is placed on top of the bed attached to the ceiling and rest are placed on the walls in a manner that they illuminate the area near the bed and the chair as shown in Fig. 3a Three RFID antennas were used in Roomset2 configuration where two of them were placed on top of the bed (ceiling) to provide better illumination of the sensor tag while the participant is getting out of the bed and the other was placed on the wall in front of the chair.

Fourteen older volunteers (age: $78 \pm 4.9$ years) were trialled; each wore a $\mathrm{W}^{2} \mathrm{ISP}$ on top of their garment at the sternum level as shown in Fig.1. Placing the sensor at the sternum level allows us to capture trunk movements, which is important to recognize aforementioned activities. Each participant was asked to perform a series of broadly scripted activity routines that were sequential arrangement of the activities: i) lying on bed; ii) sitting on bed; iii) sitting in an arm chair; and iv) walking from A to B where A, B is bed, chair or door. A hopitalized patient can only perform limited set of activities. For example, a patient may have their meal while sitting on the bed or chair or they may move about the room. Therefore, these activities are fairly comprehensive for older patient monitoring settings. Since all of these activities are important and no other activities were included in activity scripts, the 'Null' (other) activity label was not considered. These activities were observed and recorded along with the ground truth by a researcher using an in-house developed software. During the data collection patients were not using blankets, but presence of blankets will not hinder the sensor tag energy harvesting and consequently the data collection because textile materials are not radio frequency opaque.

Table 1 shows the distribution of activities in two data sets. Class labels in both data sets are unevenly distributed and this imbalance nature is more prominent in Roomset 2 as nearly $90 \%$ of the sensor observations are from lying on bed. Both data sets depict considerable variations in the sam- 


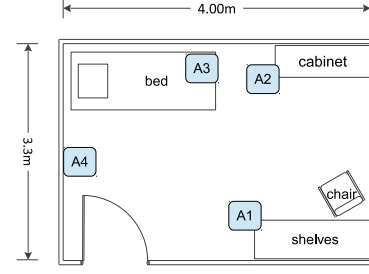

(a) Roomset1

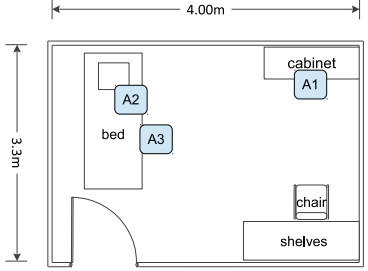

(b) Roomset2
Figure 3: Configurations of the antenna placements for the two clinical rooms used to collect data

pling rate. For Roomset1 and Roomset2 mean time differences between samples are $0.368 \pm 2.438 s$ and $0.720 \pm 9.717 s$ respectively.

\subsection{Sensor Data Stream Interpolation}

We denote the $i^{t h}$ sensor observation $s_{i}$ at time $t_{i}$, where $s_{i}$ is the 5-tuple obtained for each sensor observation and $\forall i t_{i}>t_{i-1}, i \in \mathbb{N}$, using the pair $\left(t_{i}, s_{i}\right)$ then sequence of received sensor observations $\left\{\left(t_{i}, s_{i}\right)\right\}_{i \geq 1}, i \in \mathbb{N}$ is clearly a non-uniform time series. We segment the data stream in real-time as a single sensor observation is inadequate to obtain sufficient amount of information related to a wearer's movements or current activity 21,34. In this study we utilize a fixed time sliding window approach to segment the data stream since this approach performs better than fixed sample sliding window as shown in our previous research 34. Let $\delta t$ be the segment size, then, a segment is obtained for each sensor observation, $\left(t_{i}, s_{i}\right)$, where all the sensor observations from $t_{i}-\delta t$ to $t_{i},\left\{\left(t_{k}, s_{k}\right)\right\}_{t_{k}>t_{i}-\delta t}$, are considered as the segment for $\left(t_{i}, s_{i}\right)$.

We interpolate the sensor observations within a given segment to obtain a data stream segment with a regular sampling rate. However, interpolating between sensor observations that are temporally distant would lead to large interpolation errors due to the unavailability of sufficient information to approximate the acceleration signal as indicated by large temporal gaps in our data sets (see Section 3.2). For example, consider a function $f:[a, b] \mapsto \mathbb{R}$, where $[a, b]$ denotes a bounded interval in $\mathbb{R}$, approximated by an $n^{\text {th }}$ order polynomial interpolant $P$ using a distinct set of $n+1$ data points $D=\left\{x_{0} \ldots x_{n}\right\} \subset[a, b]$. Then the interpolation error $E_{n}^{f}(x)=f\left[x_{0} \ldots x_{n}\right] \prod_{j=0}^{n}\left(x-x_{j}\right)$, where $f\left[x_{0} \ldots x_{n}\right]$ represents the $n^{t h}$ order divided difference 39 . $E_{n}^{f}(x)$ clearly indicates that interpolation error increase with $\left(x_{j+1}-x_{j}\right)$. Consequently, interpolating between sensor observations that are temporally distant leads to extracting poor contextual information related to the current activity.

To overcome this issue we use the dynamic sensor data augmentation algorithm in Algorithm 1 that considers a sequence of sensor observations $\left\{\left(t_{k}, s_{k}\right)\right\}_{t_{k}>t_{i}-2 \delta t}$ to interpolate the $i^{\text {th }}$ segment. Different interpolants require different number of minimum data points, $N$, for successful interpolation. If the required number of sensor observations for interpolation are not found (line 4 in Algorithm 1), then we augment the sensor data stream by replicating the furthest sensor observation $\left(t_{s}, s_{s}\right)$ from $\left(t_{i}, s_{i}\right)$, where $t_{s} \leq t_{i}$, at time

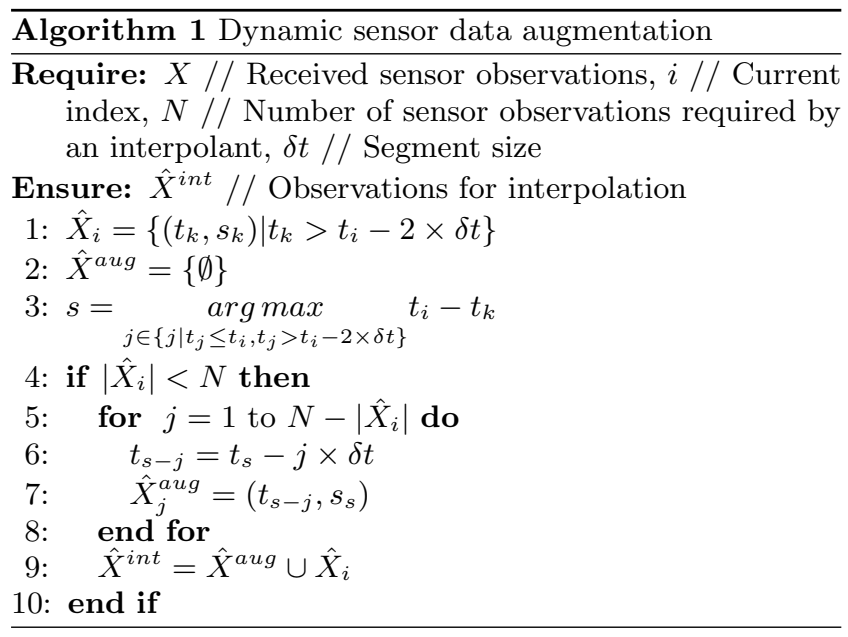

Table 2: Summary of interpolation methods used

\begin{tabular}{ll}
\hline Pre-processing & Notation \\
\hline Raw signal & Raw \\
Linear interpolation & Lin \\
Cosine interpolation & Cos \\
Cubic interpolation & Cub1 \\
Cubic convolution interpolation & Cub1 \\
Lagrange interpolation & Lag \\
\hline
\end{tabular}

steps of $\delta t$ from $t_{s}$ until the required number of samples for interpolation is obtained (line 5 in Algorithm 1). For example, if a single sensor observation needs to be augmented, then it is augmented as $\left(t_{s-1}, s_{s-1}\right)=\left(t_{s}-\delta t, s_{s}\right)$.

In this study we considered piecewise interpolation using five interpolation methods summarised in Table 2, Fig.4 illustrates an example resultant signal when aforementioned interpolation methods are applied to a 1-D sparse acceleration data stream.

We selected these interpolants mainly considering the complexity and the number of data points required for interpolation. Lower number of data points result in a poor approximation but these methods are less affected by large sampling

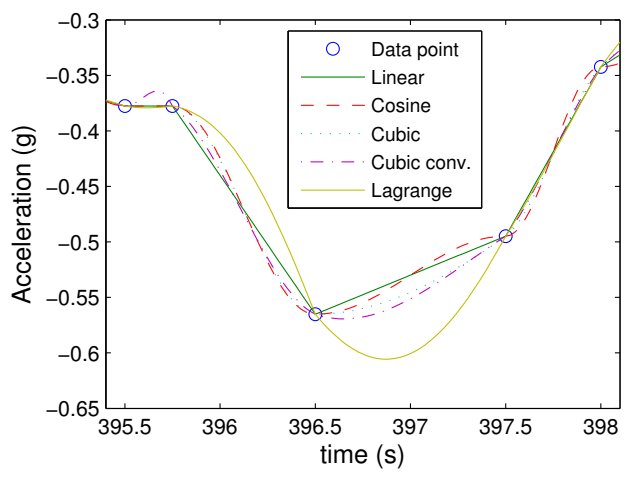

Figure 4: Segment of the $a_{f}$ acceleration signal from $\mathrm{W}^{2}$ ISP interpolated to achieve $40 \mathrm{~Hz}$ sampling rate using different interpolation methods 
intervals. In contrast, higher number of data points generally provide better approximation of a signal, but these methods are more influenced by the large sampling intervals as in our case. Both linear and cosine interpolants require two data points. In contrast to linear interpolation method, cosine interpolation method produces a smoother signal as shown in Fig. 4 Cubic and fourth order Lagrange polynomial interpolants require 4 and 5 data points respectively. Cubic convolution [19] interpolation method, which was initially proposed for image data interpolation only requires 3 data points and produces a signal similar to the cubic interpolation method (see Fig. 4 ) but with one less data point.

Application of these different interpolants produce different acceleration data streams, which we subsequently use to extract features for activity recognition. Given a segment of size $\delta t$, the interpolation methods ensure that there is a constant number of samples for each segment that allows us to extract features used in previous activity recognition studies using acceleration data.

\subsection{Features}

In this study, we considered three sets of features: i) timedomain biomechanical features from raw acceleration data $(A C C)$; ii) features based on information available from the RFID platform (RFID); and iii) features based on interpolated acceleration data $(I N T)$. We have selected common set of features successfully used in previous research and these features were exacted after segmenting data streams as discussed in Section 3.3 .

Time-domain biomechanical features: We utilized acceleration along the three axes $a_{f}, a_{l}$ and $a_{v}$ which are directly available from the sensor as features. Furthermore, trunk tilt angle about the lateral axes $\theta$ (measured on the sagittal plane) [25 28,34 and trunk tilt angle about frontal axes $\alpha$ (measured on coronal plane) can be approximated considering the acceleration signals from the $\mathrm{W}^{2} \mathrm{ISP}$ and acceleration due to gravity as $\theta_{i} \approx \tan ^{-1}\left(a_{f} / \sqrt{a_{l}^{2}+a_{v}^{2}}\right)$ and $\alpha_{i} \approx \tan ^{-1}\left(a_{l} / a_{v}\right)$. We also obtained change in vertical velocity $\left(\Delta v_{z}\right)$ and vertical displacement $\left(\Delta d_{z}\right)$ by integrating the acceleration signal $a_{z}$ along the vertical direction after adjusting for gravity with respect to a sensor tag wearer 11]. Although $\Delta v_{z}$ and $\Delta d_{z}$ can be calculated from raw sensor data, their values are influenced by interpolation because of the differences in acceleration signal from different interpolants as shown in Fig. 4. In total, we considered a set of 7 features from raw acceleration signal $(A C C)$.

RFID platform based features: The RFID platform reports $R S S I$ for a sensor observation and the identifier of the antenna $(a I D)$ that received the reading. Therefore, $R S S I$ and $a I D$ were used as features. Furthermore, we also utilized a set of features to incorporate activity contextual information that have been used previously 34 (specifically MI2 approach in 34). These features were extracted based on the antennas that collected the sensor observations pertaining to a given segment; hence the resulting feature is a vector $M I 2 \in \mathbb{R}^{\mathcal{N}_{\mathcal{A}}}$ where $\mathcal{N}_{\mathcal{A}}$ is the number of antennas used for obtaining sensor observations. Furthermore, RSSI is hyper sensitive to distance, $d$, of the sensor tag to the antenna that collected data since $R S S I \propto 1 / d^{4} 18$. Therefore, this set of features (RFID) based on the information from the RFID platform dependents on the antenna deployment and provides information regarding the approximate location (contextual information) of the sensor tag and consequently the sensor tag wearer.

Features based on interpolated acceleration: We extracted following features that require a signal with a regular sampling rate from interpolated acceleration signals (INT). Based on the three acceleration signals, $a_{f}, a_{l}, a_{v}$, and the resultant acceleration signal, $a_{r}=\sqrt{a_{f}^{2}+a_{l}^{2}+a_{v}^{2}}$, we extracted: i) 80 FFT coefficients 24]; ii) energy (squared sum of FFT coefficients) 3 30, and iii) spectral density by considering the first 10 FFT coefficients excluding the DC component (9 features) [2. Furthermore, we have also used coefficients of a fourth order auto regressive model ( 5 features) for the three acceleration signals $a_{f}, a_{l}$, and $a_{v}$ as it has been used successfully in 20. Thus, we obtain 375 additional features $(I N T)$ that are only available if interpolated acceleration signals are used.

\section{EXPERIMENTS AND RESULTS}

In this study we mainly evaluate AR performance when different interpolation methods summarised in Table 2 are used to condition the sparse data streams from a sensor tag prior to feature extraction. Our main aim is to identify whether the additional features extracted using the interpolated data stream $(A C C \cup R F I D \cup I N T)$ achieve a significant performance improvement over the limited number of features possible from a raw sensor data stream $(A C C \cup R F I D)$ in the context of body-worn passive RFID sensors. We also evaluate activity recognition performance using features calculated based on data from the accelerometer $(A C C)$ and features based on information from the RFID platform $(R F I D)$. Finally, we evaluate the mean time taken to generate a feature vector when interpolation is utilized as a measure of cost involved in additional processing (see Table 2).

We utilized two state of the art discriminative classification algorithms successfully used in previous activity recognition studies: i) Support Vector Machine (SVM) 38]; and ii) Conditional Random Fields (CRF) 33. We utilized linear SVM $\left(\mathrm{SVM}^{\text {lin }}\right)$ 7, non-linear SVM using Radial Basis Function (RBF) kernel $\left(\mathrm{SVM}^{r b f}\right)$ 6 and the linear chain CRF implementation proposed in 34 because we are interested in applications that require real-time AR. During experiments, we trained activity recognition models based on $\mathrm{SVM}^{l i n}$, $\mathrm{SVM}^{r b f}$ and CRF to recognize activities: i) sitting on bed; ii) lying on bed; iii) ambulating; and iv) sitting on chair using the collected data sets (see Section 3.2.

As shown in our previous research 34, segment size influences classification performance mainly through contextual features calculated based on a segment. Segment size also influence the feature extraction performance as it increases the number of data points to be processed. Therefore, we also evaluate different segment sizes for feature extraction and different interpolation methods.

\subsection{Activity Recognition Performance}

We evaluated AR performance based on the mean F-score for multi-class classification. F-score is the harmonic mean of the metrics precision $(\mathrm{P})$ and recall $(\mathrm{R})$ and is calculated 


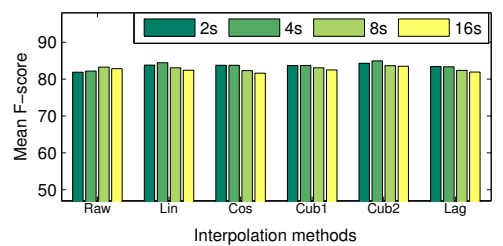

(a) $\mathrm{SVM}^{\text {lin }}$

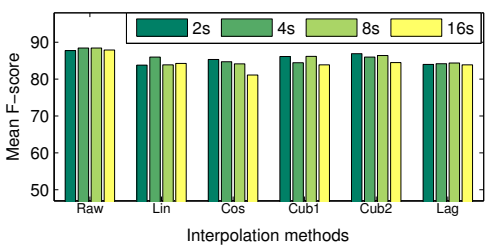

(b) $\mathrm{SVM}^{r b f}$

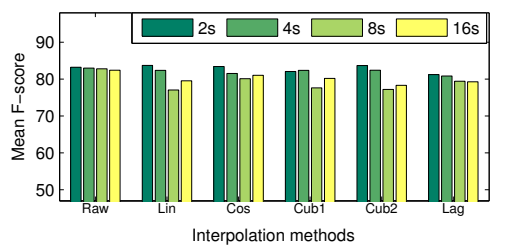

(c) $\mathrm{CRF}$

Figure 5: Real-time activity recognition performance for Roomset1 configuration (Raw: $A C C \cup R F I D$; other interpolated methods: $A C C \cup R F I D \cup I N T$ )

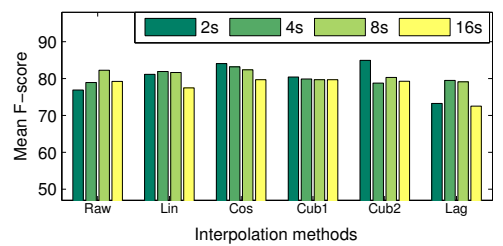

(a) $\mathrm{SVM}^{\text {in }}$

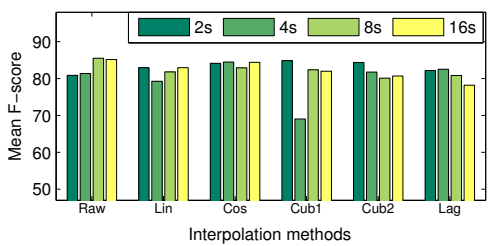

(b) $\mathrm{SVM}^{r b f}$

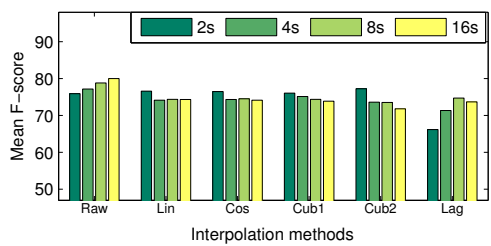

(c) $\mathrm{CRF}$

Figure 6: Real-time activity recognition performance for Roomset2 configuration (Raw: $A C C \cup R F I D$; other interpolated methods: $A C C \cup R F I D \cup I N T$ )

Table 3: Activity recognition performance for both data sets*

\begin{tabular}{llll}
\hline \multicolumn{2}{c}{ SVM $^{\text {lin }}$} & SVM $^{\text {rbf }}$ & CRF \\
\hline Roomset1 & & \\
\hline Raw & $83.27 \pm 2.37(8)$ & $\mathbf{8 8 . 4 5} \pm \mathbf{1 . 6 8}(8)$ & $83.22 \pm 3.52(2)$ \\
\hline Lin & $84.44 \pm 1.90(4)$ & $85.98 \pm 1.25(4)$ & $\mathbf{8 3 . 7 3} \pm 2.40(2)$ \\
Cos & $83.78 \pm 1.09(4)$ & $85.33 \pm 3.69(2)$ & $83.42 \pm 2.21(2)$ \\
Cub1 & $83.70 \pm 3.41(2)$ & $86.17 \pm 1.83(8)$ & $82.39 \pm 2.90(4)$ \\
Cub2 & $\mathbf{8 4 . 9 6} \pm \mathbf{1 . 2 3 ( 4 )}$ & $86.91 \pm 3.00(2)$ & $83.67 \pm 1.89(2)$ \\
Lag & $83.43 \pm 1.57(2)$ & $84.39 \pm 2.19(8)$ & $81.25 \pm 2.66(2)$ \\
\hline Roomset2 & & \\
\hline Raw & $82.28 \pm 3.04(8)$ & $\mathbf{8 5 . 5 3} \pm \mathbf{2 . 8 6}(8)$ & $\mathbf{7 9 . 9 9} \pm \mathbf{4 . 7 6}(16)$ \\
\hline Lin & $81.92 \pm 4.32(4)$ & $82.97 \pm 3.02(16)$ & $76.61 \pm 4.24(2)$ \\
Cos & $84.05 \pm 6.21(2)$ & $84.48 \pm 2.76(4)$ & $76.48 \pm 3.95(2)$ \\
Cub1 & $80.42 \pm 6.27(2)$ & $84.86 \pm 3.57(2)$ & $76.08 \pm 6.21(2)$ \\
Cub2 & $\mathbf{8 4 . 9 7} \pm \mathbf{3 . 4 7}(2)$ & $84.36 \pm 3.34(2)$ & $77.26 \pm 3.62(2)$ \\
Lag & $79.52 \pm 4.33(4)$ & $82.56 \pm 5.90(4)$ & $74.70 \pm 2.83(2)$ \\
\hline
\end{tabular}

Results present $\mathrm{F}$-score [mean $\pm \mathrm{SD}]$; the segment size $\delta t$ for each value is shown in brackets; highest mean performance for each classifier and room configuration is shown in bold face; highest mean performance for each classifier for interpolation methods are shown in italics.

as $F$-score $=2 . P . R /(P+R)$ where precision $(\mathrm{P})$ and recall $(\mathrm{R})$ are used as per the standard definitions. In contrast to accuracy, F-score provides a better view of the classifier performance, specially for data sets with imbalanced class distributions as in our case (see Table 1), because F-score is not biased towards the majority class [14]. We also present our final results using accuracy, precision, recall and specificity for completeness.

We obtained activity recognition performance using a 10fold cross validation strategy. We placed each trial (a contin- uous recording of a broadly scripted activity sequence from a single participant) randomly in tandem. Then we subdivided the data set into 10 portions (folds) where each fold constitutes complete activity sequences as required by the CRF classifier. Our model selection strategy was to select the model with highest $\mathrm{F}$-score for validation.

We initially evaluated activity recognition performance for features possible form raw data stream and features possible from interpolated acceleration signal. Then we further evaluated activity performance based on different feature sets.

\subsubsection{Raw and interpolated acceleration data fea- tures}

We obtained activity recognition performance for each interpolant (see Table 2 based on segment sizes $\delta t \in\{2,4,8,16\}$ for each classifier. Figure 5 and Fig. 6 present activity recognition performance for the two data sets with the three classification models. Table 3 presents the highest performing classifiers for each interpolation method for both data sets, i.e. Roomset1 and Roomset2. Raw is used with features $A C C \cup R F I D$ which can be obtained without interpolation. Activity recognition performance for the interpolants were obtained using features $A C C \cup R F I D \cup I N T$. From these results we can observe that, in 4 out of 6 instances, performance (i.e. mean F-score) of Cub2 is higher but statistically insignificant compared to other interpolation methods (excluding Raw). This general consistency is because the Cub2 interpolant is able to provide better approximation for acceleration signals when compared to Lin and Cos interpolants. Furthermore, noisy acceleration data have a lower influence on $C u b 2$ compared to $C u b 1$ and $L a g$ because $C u b 2$ require lower number of data points $(N=3)$ with respect to $C u b 1(N=4)$ and $\operatorname{Lag}(N=5)$ for interpolation. Given the sparse nature of the data stream, interpolants that require more number of data points such as Cub1 and Lag 
Table 4: Best activity recognition performances (mean F-score) for each classification model.

\begin{tabular}{|c|c|c|c|c|c|c|}
\hline & $\begin{array}{l}\text { Interpolant } \\
(\delta t)^{\mathrm{a}}\end{array}$ & $\begin{array}{l}\text { Accuracy } \\
(\text { mean } \pm S D)\end{array}$ & $\begin{array}{l}\text { Precision } \\
(\text { mean } \pm \text { SD })\end{array}$ & $\begin{array}{l}\text { Recall } \\
(\text { mean } \pm \mathrm{SD})\end{array}$ & $\begin{array}{l}\text { Specificity } \\
(\text { mean } \pm \text { SD })\end{array}$ & $\begin{array}{l}\text { F-score } \\
(\text { mean } \pm S D)\end{array}$ \\
\hline \multicolumn{7}{|c|}{ Roomset1 } \\
\hline $\mathrm{SVM}^{\text {lin }}$ & $C u b 2(4 \mathrm{~s})$ & $98.00 \pm 0.41$ & $87.87 \pm 2.55$ & $83.44 \pm 1.72$ & $98.61 \pm 0.30$ & $84.96 \pm 1.23$ \\
\hline $\mathrm{SVM}^{r b f}$ & $\operatorname{Raw}(8 \mathrm{~s})$ & $98.43 \pm 0.41$ & $90.39 \pm 2.70$ & $87.42 \pm 1.42$ & $98.82 \pm 0.47$ & $88.45 \pm 1.68$ \\
\hline $\mathrm{CRF}$ & $\operatorname{Lin}(2 \mathrm{~s})$ & $97.28 \pm 0.39$ & $85.97 \pm 2.43$ & $82.35 \pm 3.08$ & $97.94 \pm 0.22$ & $83.73 \pm 2.40$ \\
\hline \multicolumn{7}{|c|}{ Roomset2 } \\
\hline $\mathrm{SVM}^{\text {lin }}$ & $C u b 2(2 \mathrm{~s})$ & $99.09 \pm 0.47$ & $87.06 \pm 4.10$ & $84.00 \pm 2.90$ & $98.70 \pm 0.56$ & $84.97 \pm 3.74$ \\
\hline $\mathrm{SVM}^{r b f}$ & $\operatorname{Raw}(8 \mathrm{~s})$ & $99.18 \pm 0.34$ & $90.97 \pm 4.11$ & $83.88 \pm 2.04$ & $98.55 \pm 0.79$ & $85.53 \pm 2.86$ \\
\hline $\mathrm{CRF}$ & $\operatorname{Raw}(16 \mathrm{~s})$ & $98.37 \pm 0.58$ & $83.68 \pm 6.50$ & $78.29 \pm 3.58$ & $96.23 \pm 1.12$ & $79.99 \pm 4.76$ \\
\hline
\end{tabular}

${ }^{\text {a }}$ Corresponding segment sizes that yield the best performance is given in brackets next to preprocessing methods

${ }^{*}$ Model parameters - SVM ${ }^{\text {lin }}:\left[\right.$ Roomset $1: c=2^{0} ;$ Roomset $\left.2: c=2^{5}\right] ; \operatorname{SVM}^{\text {rbf }}:[$ Roomset1: $c=$ $2^{5}, \gamma=2^{-1} ;$ Roomset2: $\left.\left.c=2^{9}, \gamma=2^{-7}\right]\right)$; and CRF: [Roomset $1: \lambda=8.5 ;$ Roomset $\left.2: \lambda=1.6\right]$

result in inclusion of temporaly distant sensor observations for successful interpolation (see Section 3.3). This also results in poor approximations of missing acceleration data as the temporaly distant observations are most likely to belong associated with different activities. Therefore, $C u b 2$ is able to produce more informative features for AR. On the other hand Lag depicted the lowest performance among all the interpolants. The main reason is that Lag is more influenced, compared to others, by data stream sparsity as it requires five data points. Additionally, if number of data points, $N$, required for interpolation is $N<5$, then sensor observations are augmented as stated in the algorithm in Algorithm 1 . Although augmenting sensor observations is necessary to reduce interpolation errors, augmenting higher number of sensor observations may significantly change the activity patterns represented in acceleration data. Therefore, increasing the number of augmented sensor observations, as in the case of $L a g$, adversely affect extracted features and subsequently results in further decreasing AR performance.

From Fig. 5 and Fig.6 and also from Table 3 , it is evident that different classification models responded differently to the interpolated data streams. $\mathrm{SVM}^{\text {lin }}$ was able to achieve performance improvements of $2 \%$ for Roomset 1 and $3 \%$ for Roomset2 with interpolated data. CRF only depicted a $0.6 \%$ performance improvement for Roomset 1 . In the case of SVM ${ }^{r b f}$, although interpolation has increased the performance for segment sizes $\delta t=2 s$ and $\delta t=8 s$ for Roomset2 data set (see Fig.6), but in general, interpolation has degraded the performance in $\mathrm{SVM}^{r b f}$. This is mainly due to the use of RBF kernel in $\mathrm{SVM}^{r b f}$ which is more sensitive to noise in the features. This feature noise is mainly attributed by the noise inherent in the acceleration data. Since acceleration data is used to calculate additional features, the noise in the acceleration data have a greater influence on interpolated features. Although our data stets are imbalanced, F-score for all the classes depicted good recognition performance.

Table 4 presents best activity recognition performances achieved by each classification model. From Table 4 , it is evident that $\mathrm{SVM}^{r b f}$ classifier with raw acceleration signals has outperformed (mean F-score) the other activity classification models for both data sets. Although improvements in mean performance was obtained using additional features possible with interpolated data $(I N T)$, no statistically significant improvement was observed, therefore, we further investigate activity recognition performance based on different feature sets: i) time-domain biomechanical features from raw acceleration data $(A C C)$; ii) features based on information from the RFID platform ( $R F I D)$; iii) featured possible from the raw data stream $(A C C \cup R F I D)$; and iv) raw acceleration based time-domain biomechanical features and features from interpolated acceleration signals using best interpolation methods (based on mean F-Score) for each classifier (see Table 3) $(A C C \cup I N T)$.

\subsubsection{Comparing performance of feature sets}

Fig.7 and Fig.8 illustrate AR performance for feature sets $A C C, R F I D, A C C \cup R F I D$ and $A C C \cup I N T$ for Roomset 1 and Roomset2 respectively. It is interesting to note that, when features from acceleration signals are compared, i.e. $A C C$ and $A C C \cup I N T$, using interpolated acceleration signals have significantly $(p<0.05)$ improved AR performance across all classifiers. This performance improvement is hidden in Fig. 5 and 6. because as illustrated in Fig.7 and Fig. 8 incorporating the feature set RFID with $A C C$ also depicted a significant performance improvement over $A C C$ feature set for all classifiers. This clearly indicates that RFID complements $A C C$ similarly to $I N T$ for obtaining contextual information for $\mathrm{AR}$ and hence we can successfully recognize activities without features not readily obtained from sparse acceleration data.

When activity recognition performance using feature sets that include $I N T$ are considered, in general, activity recognition performance has decreased with segment size $\delta t$; segment size $\delta t=2 s$ has outperformed other segment sizes (see Fig. 5, 6, 7 and 8). Increasing the segment size results in sensor observations from previous activities to be considered for interpolation resulting extraction of less discriminative values for features. We can observe a similar pattern when the $A C C$ feature set is considered, mainly due to the incorrect values assumed for the features $\Delta v_{z}$ and $\Delta d_{z}$ as they are approximated by integrating the acceleration signal over the segment.

However, increasing $\delta t$ has a lower influence on the $R F I D$ 


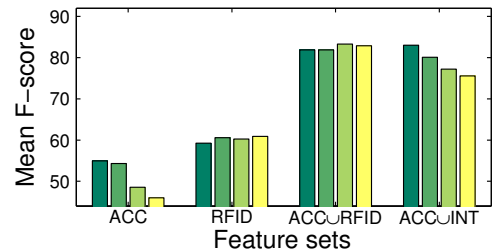

(a) SVM ${ }^{\text {lin }}$

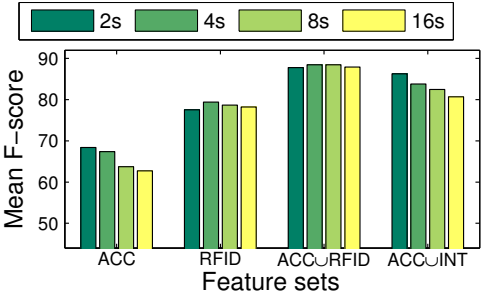

(b) $\mathrm{SVM}^{r b f}$

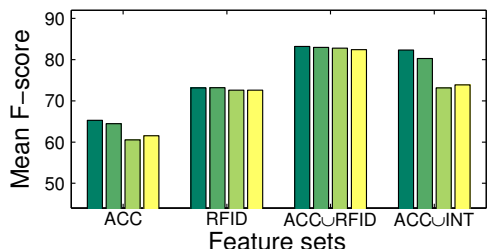

(c) CRF

Figure 7: Roomset1 real-time activity recognition performance for different feature sets ( $A C C$ : features from raw acceleration; RSSI: features from RSSI; INT: features from interpolated acceleration signal). Interpolation methods used for $\mathbf{S V M}^{l i n}: C u b 2 ; \mathbf{S V M}^{r b f}: C u b 2 ; \mathbf{C R F}:$ Lin

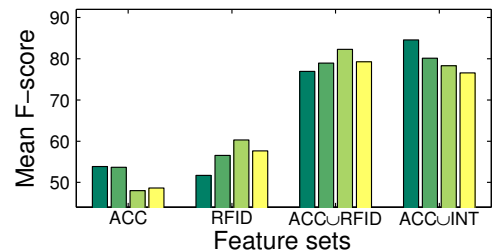

(a) SVM ${ }^{\text {lin }}$

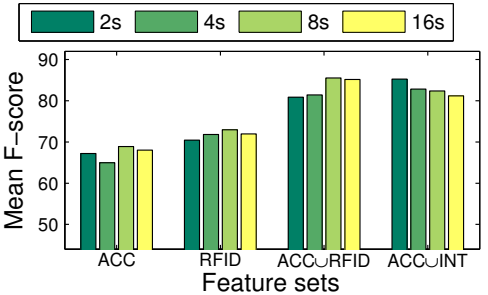

(b) $\mathrm{SVM}^{r b f}$

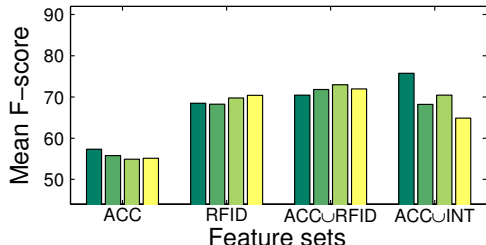

(c) CRF

Figure 8: Roomset2 real-time activity recognition performance for different feature sets (ACC: features from raw acceleration; RSSI: features from RSSI; INT: features from interpolated acceleration signal). Interpolation methods used for $\mathbf{S V M}^{l i n}: C u b 2 ; \mathbf{S V M}^{r b f}: C u b 1 ; \mathbf{C R F}: C u b 2$

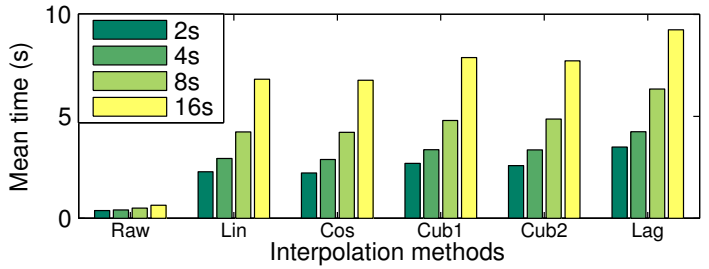

Figure 9: Mean time taken to extract a feature vector using a single segment for each preprocessing method in Table 2 for different segment sizes.

feature set as the feature $M I 2$, which is calculated based on the segment, is not influenced by the inter sensor observation time intervals (i.e. sparsity) as with feature sets $A C C$ and $A C C \cup I N T$. In general, optimum segment size that results in more discriminative activity recognition models for features based on acceleration data $(A C C$ and $A C C \cup I N T)$ is different to that of features based on the information from RFID platform $(R F I D)$. Therefore, when the same segment size is used to extract all the features $(A C C \cup R F I D \cup$ $I N T)$, overall performance decreases. This has largely influenced the $\mathrm{SVM}^{r b f}$ classifier depicting a performance decrease when using all the features $(A C C \cup R F I D \cup I N T)$ (see Fig. 5 and 6). This indicates that using different segment sizes to extract features from these different information sources may result in activity prediction models with higher performance.

\subsection{Interpolation Cost}

Time taken for interpolating the acceleration data stream and extracting additional features can be viewed as the cost incurred to obtain an improvement in activity recognition performance. We considered the time taken to interpolate and extract features together in contrast to treating them separately because interpolation is required to condition the data stream before extracting additional features (see Section 3.4.

We obtained mean time to generate a feature vector for a single segment based on different segment sizes because the number of data points to be considered for feature extraction increases linearly with segment size. We have investigated the interpolation methods summarized in Table 2, Results in Fig.9 are obtained using Matlab (version: 8.2.0.701) on a machine running Windows 8.1 operating system with 8 GB Random Access Memory and x-64 processor with 4 cores running at $2.40 \mathrm{GHz}$ (Intel Core i7-3630QM @ $2.4 \mathrm{GHz}$ ).

Fig.9 shows the mean time taken to generate a feature vector for a single segment using each interpolation method in Table 2 As expected, time taken to generate a feature vector using interpolated acceleration is at least 5 folds greater than that of using Raw data. Furthermore, increasing the segment size linearly increases the mean time for generating a feature vector and this is associated with the linear increase in samples with the segment size.

These results (see Fig.9 also depicts that the mean time taken by each interpolant depends on the complexity of the interpolant. For example, Lag showed the highest mean time taken as it required the most number of data points, i.e. $N=5$. 


\section{CONCLUSIONS}

In this study, we evaluated the use of online interpolation for real-time activity recognition using sparse and noisy acceleration data streams from a wearable passive RFID sensor. Online interpolation enabled readily computing features requiring data streams with regular sampling rates.

There are two main findings from this study. Firstly, when only acceleration from sensor tags are considered, features obtained using interpolation significantly boost the activity recognition performance compared to time-domain biomecahincal features from raw acceleration data. Secondly, features based on information from an RFID platform can also significantly improve activity recognition performance and achieve comparable results to activity recognition models built using additional features possible from interpolation. This indicates that features from the RFID platform can be successfully substituted to replace the additional features, such as FFT based features, possible from interpolated acceleration data to achieve similar or better activity recognition performance. On the other hand, RFID deployment agnostic activity recognition models can be learnt using features based only on acceleration data while enjoying the advantages provided by sensor tags but with a considerable real-time prediction delay ( $>400 \%$ compared to Raw).

There are several future directions to this study. Firstly, we have only considered engineered features that were successfully used in previous AR research but feature learning 4 27] with interpolated acceleration data from passive sensors to further improve AR performance should be considered in future. Secondly, we have only utilized two data sets with a limited number of activities related to ambulatory monitoring in the context of monitoring older people in hospital and nursing homes. Therefore, further investigation using sparse data streams when such data sets become available will be needed in future to further generalize the results we have presented. Finally, the investigated feature sets depicted their respective highest performances for different segment sizes as shown in Fig.7 and Fig.8 Therefore, we expect to observe an improvement in activity recognition performance when using $A C C \cup R F I D \cup I N T$ with features extracted using the respective segment sizes that resulted in the highest performance. This also needs to be further investigated in future.

In conclusion, this study provides a basis for many ubiquitous research and applications based on real-time activity recognition using wearable passive sensors, especially passive sensors enabled RFID tags.

\section{ACKNOWLEDGMENTS}

This research was supported by a grant from the Department of State Development (DSD) under the Collaboration Pathways Program and the Australian Research Council (DP130104614).

\section{REFERENCES}

[1] L. Atallah, B. Lo, R. King, and G.-Z. Yang. Sensor positioning for activity recognition using wearable accelerometers. IEEE Trans. Biomed. Circuits Syst., $5(4): 320-329,2011$.
[2] O. Banos, M. Damas, H. Pomares, A. Prieto, and I. Rojas. Daily living activity recognition based on statistical feature quality group selection. Expert Syst. Appl., 39(9):8013-8021, 2012.

[3] L. Bao and S. S. Intille. Activity recognition from user-annotated acceleration data. In A. Ferscha and F. Mattern, editors, Pervasive Computing, number 3001 in Lecture Notes in Computer Science, pages 1-17. Springer Berlin Heidelberg, 2004.

[4] S. Bhattacharya, P. Nurmi, N. Hammerla, and T. Plötz. Using unlabeled data in a sparse-coding framework for human activity recognition. arXiv:1312.6995 [cs, stat], 2013.

[5] M. Buettner, B. Greenstein, A. Sample, J. R. Smith, and D. Wetherall. Revisiting smart dust with RFID sensor networks. In Proceedings of the 7th ACM Workshop on Hot Topics in Networks (HotNets-VII), 2008.

[6] C.-C. Chang and C.-J. Lin. LIBSVM: A library for support vector machines. ACM Trans. Intell. Syst. Technol., 2(3):27:1-27:27, 2011.

[7] R.-E. Fan, K.-W. Chang, C.-J. Hsieh, X.-R. Wang, and C.-J. Lin. LIBLINEAR: A library for large linear classification. J. Mach. Learn. Res., 9:1871-1874, 2008.

[8] J. Fessler and B. Sutton. Nonuniform fast fourier transforms using min-max interpolation. IEEE Trans. Signal Process., 51(2):560-574, 2003.

[9] L. Gao, A. K. Bourke, and J. Nelson. Sensor positioning for activity recognition using multiple accelerometer-based sensors. In European Symposium on Artificial Neural Networks, Computational Intelligence and Machine Learning (ESANN), 2013.

[10] L. Gao, A. K. Bourke, and J. Nelson. Evaluation of accelerometer based multi-sensor versus single-sensor activity recognition systems. Med. Eng. Phys., 36(6):779-785, 2014.

[11] A. Godfrey, A. Bourke, G. Ólaighin, P. van de Ven, and J. Nelson. Activity classification using a single chest mounted tri-axial accelerometer. Med. Eng. Phys., 33(9):1127-1135, 2011.

[12] L. Greengard and J. Lee. Accelerating the nonuniform fast fourier transform. SIAM Review, 46(3):443-454, 2004.

[13] M. Gövercin, Y. Költzsch, M. Meis, S. Wegel, M. Gietzelt, J. Spehr, S. Winkelbach, M. Marschollek, and E. Steinhagen-Thiessen. Defining the user requirements for wearable and optical fall prediction and fall detection devices for home use. Inform. Health Soc. Care, 35(3-4):177-187, 2010.

[14] H. He and E. Garcia. Learning from imbalanced data. IEEE Trans. Knowl. Data Eng., 21(9):1263-1284, 2009.

[15] Z. He and L. JIN. Activity recognition from acceleration data based on discrete consine transform and SVM. In IEEE International Conference on Systems, Man and Cybernetics, pages 5041-5044, 2009.

[16] H. Junker, O. Amft, P. Lukowicz, and G. Tröster. Gesture spotting with body-worn inertial sensors to detect user activities. Pattern Recognition, 41(6):2010-2024, 2008. 
[17] D. Karantonis, M. Narayanan, M. Mathie, N. Lovell, and B. Celler. Implementation of a real-time human movement classifier using a triaxial accelerometer for ambulatory monitoring. IEEE Trans. Inf. Technol. Biomed., 10(1):156-167, 2006.

[18] T. Kaufmann, D. C. Ranasinghe, M. Zhou, and C. Fumeaux. Wearable quarter-wave folded microstrip antenna for passive UHF RFID applications. Int. J. Antennas Propag., 2013, 2013.

[19] R. Keys. Cubic convolution interpolation for digital image processing. IEEE Transactions on Acoustics, Speech and Signal Processing, 29(6):1153-1160, 1981.

[20] A. M. Khan, A. Tufail, A. M. Khattak, and T. H. Laine. Activity recognition on smartphones via sensor-fusion and KDA-based SVMs. Int. J. Distrib. Sens. Netw., 2014:e503291, 2014.

[21] N. C. Krishnan and D. J. Cook. Activity recognition on streaming sensor data. Pervasive and Mobile Computing, 2012.

[22] M.-W. Lee, A. M. Khan, and T.-S. Kim. A single tri-axial accelerometer-based real-time personal life log system capable of human activity recognition and exercise information generation. Pers. Ubiquitous Comput., 15(8):887-898, 2011.

[23] F. Miskelly. A novel system of electronic tagging in patients with dementia and wandering. Age and Ageing, 33(3):304-306, 2004.

[24] S. A. Muhammad, B. N. Klein, K. V. Laerhoven, and K. David. A feature set evaluation for activity recognition with body-worn inertial sensors. In R. Wichert, K. V. Laerhoven, and J. Gelissen, editors, Constructing Ambient Intelligence, number 277 in Communications in Computer and Information Science, pages 101-109. Springer Berlin Heidelberg, 2012.

[25] B. Najafi, K. Aminian, A. Paraschiv-Ionescu, F. Loew, C. Bula, and P. Robert. Ambulatory system for human motion analysis using a kinematic sensor: Monitoring of daily physical activity in the elderly. IEEE Trans. Biomed. Eng., 50(6):711-723, 2003.

[26] M. Patel and J. Wang. Applications, challenges, and prospective in emerging body area networking technologies. IEEE Wireless Commun. Mag., 17(1):80-88, 2010.

[27] T. Plötz, N. Y. Hammerla, and P. Olivier. Feature learning for activity recognition in ubiquitous computing. In IJCAI Proceedings-International Joint Conference on Artificial Intelligence, volume 22, page 1729, 2011.

[28] D. C. Ranasinghe, R. L. Shinmoto Torres, K. Hill, and R. Visvanathan. Low cost and batteryless sensor-enabled radio frequency identification tag based approaches to identify patient bed entry and exit posture transitions. Gait \& Posture, 39(1):118-123, 2014.

[29] D. C. Ranasinghe, R. L. Shinmoto Torres, A. P. Sample, J. R. Smith, K. Hill, and R. Visvanathan. Towards falls prevention: A wearable wireless and battery-less sensing and automatic identification tag for real time monitoring of human movements. In Annual International Conference of the IEEE Engineering in Medicine and Biology Society (EMBC), pages $6402-6405,2012$

[30] N. Ravi, N. Dandekar, P. Mysore, and M. L. Littman. Activity recognition from accelerometer data. In AAAI, pages 1541-1546, 2005.

[31] H. Saito, T. Watanabe, and A. Arifin. Ankle and knee joint angle measurements during gait with wearable sensor system for rehabilitation. In World Congress on Medical Physics and Biomedical Engineering, IFMBE Proceedings, pages 506-509. 2009.

[32] A. P. Sample, D. J. Yeager, P. S. Powledge, A. V. Mamishev, and J. R. Smith. Design of an RFID-based battery-free programmable sensing platform. IEEE Trans. Instrum. Meas., 57(11):2608-2615, 2008.

[33] R. L. Shinmoto Torres, D. C. Ranasinghe, Qinfeng Shi, and A. P. Sample. Sensor enabled wearable RFID technology for mitigating the risk of falls near beds. In IEEE International Conference on RFID, pages 191-198, 2013.

[34] R. L. Shinmoto Torres, D. C. Ranasinghe, and Q. Shi. Evaluation of wearable sensor tag data segmentation approaches for real time activity classification in elderly. In 10th International Conference on Mobile and Ubiquitous Systems: Computing, Networking and Services, 2013.

[35] Y. Su, A. Wickramasinghe, and D. C. Ranasinghe. Investigating Sensor Data Retrieval Schemes for Multi-Sensor Passive RFID Tags. In IEEE International Conference on RFID, pages 201-208, San Diego, USA, 2015.

[36] T. Tamrat, M. Griffin, S. Rupcic, S. Kachnowski, T. Taylor, and J. Barfield. Operationalizing a wireless wearable fall detection sensor for older adults. In 6th International Conference on Pervasive Computing Technologies for Healthcare (PervasiveHealth), pages 297-302, 2012

[37] D. Tao, L. Jin, Y. Yuan, and Y. Xue. Ensemble manifold rank preserving for acceleration-based human activity recognition. IEEE Trans. Neural Netw. Learn. Syst., Early Access Online, 2014.

[38] J. P. Varkey, D. Pompili, and T. A. Walls. Human motion recognition using a wireless sensor-based wearable system. Pers. Ubiquitous Comput., 16(7):897-910, 2012.

[39] J. d. Villiers. Error analysis for polynomial interpolation. In Mathematics of Approximation, number 1 in Mathematics Textbooks for Science and Engineering, pages 25-35. Atlantis Press, 2012.

[40] L. Wang, T. Gu, X. Tao, and J. Lu. A hierarchical approach to real-time activity recognition in body sensor networks. Pervasive Mob. Comput., 8(1):115-130, Feb. 2012.

[41] K.-H. Wolf, K. Hetzer, H. M. z. Schwabedissen, B. Wiese, and M. Marschollek. Development and pilot study of a bed-exit alarm based on a body-worn accelerometer. Z. Gerontol. Geriatr., 46(8):727-733, 2013.

[42] K. Zhan, S. Faux, and F. Ramos. Multi-scale conditional random fields for first-person activity recognition. In IEEE International Conference on Pervasive Computing and Communications, pages 51-59, 2014. 